\title{
Current Situation and Innovative Development of Food Quality Testing Technology in Big Data
}

\author{
Tao $\mathrm{Ji}^{1, a, *}$, Jingbo $\mathrm{Ye}^{2}$, Liangbo Wang ${ }^{1}$ \\ ${ }^{1}$ Sichuan Vocational College of Chemical Technology, Luzhou, Sichuan, 646000, China \\ ${ }^{2}$ Sichuan National Inspection and Testing Co. Ltd. Luzhou, Sichuan, 646000, China \\ a lzdszx@163.com \\ *corresponding author
}

Keywords: Big Data, Food; Quality, Detection Technology, Rfid.

\begin{abstract}
In view of the shortage of food quality and safety tracking and testing technology in our country, it is necessary to construct and perfect the food safety quality inspection system based on big data. Based on the foreign food quality and safety inspection and supervision technology, this article takes the animal source food quality assurance system as an example, constructs the intelligent, automatic quality inspection and tracking technology, uses the big data technology to carry on the data type analysis..
\end{abstract}

\section{Introduction}

With the deepening of China's reform and opening up, the development of modern science and technology and the improvement of people's living standards, people's requirements for food quality are also improving, and the production and processing channels of food are also increasing, and food quality and safety have become an important aspect to ensure people's livelihood.[1]With the factors of enterprise leading and market needing, the food production and development of our country has entered a new stage, and gradually developed into the pillar industry of national economy development. In the process of food production and development, in addition to the urgent need to improve the efficiency of food production, but also faced with increasingly severe food safety adjustment. In developed countries, the European Union, Japan, the United States and other developed countries have witnessed various food production safety incidents, such as foot-andmouth disease, mad cow disease and other malignant food safety incidents, these food safety incidents have brought very serious public health safety. These food safety problems also have a very bad impact on the food market, which makes the image of the whole industry seriously damaged, reduces the competitiveness of the industry, and threatens the benefits and development of the food industry.[2]With the continuous development of Internet technology, the use of big data technology can achieve intelligent and safe production of food, the whole process of recording and understanding of food production, processing, transportation process, to achieve the whole process of food backtracking management. This study takes the management of animal food safety production as the research object to understand the application of big data in food quality inspection and safety management.

\section{Analysis of Current Situation of Food Safety Management}

\subsection{Improper Use of Additives in Food Production}

In the process of animal breeding, in order to avoid the occurrence of epidemic disease, people will add many veterinary drugs, use vaccines and so on, on the other hand, in order to enable animals to quickly out of the tank, can be put into the market to achieve benefits. The use of feed has been one of the essential material bases of modern animal husbandry production, which can promote the growth of animals and improve the quality of products. However, this kind of behavior 
of using veterinary drugs and additives in large quantities, although it can achieve the effect of increasing production, increasing income and treating diseases, because excessive use of veterinary drugs violates the regulations of using veterinary drugs, the residue of veterinary drugs will also cause harm to human body, which has gradually become an important factor affecting food safety.[3]The veterinary drug residue refers to the drug or the corresponding metabolic product that the animal will remain in the animal body after the use of the drug, such as eggs, meat, etc., this residue is caused by the improper use of the drug, because the farm did not use the drug in accordance with the withdrawal period or the use of prohibited drugs. It is also possible to think that the livestock that have been used in the farm are not marked in time, resulting in repeated medication. Some farms may use drugs to cover up the possible clinical symptoms of livestock in order to reduce the possible economic losses.

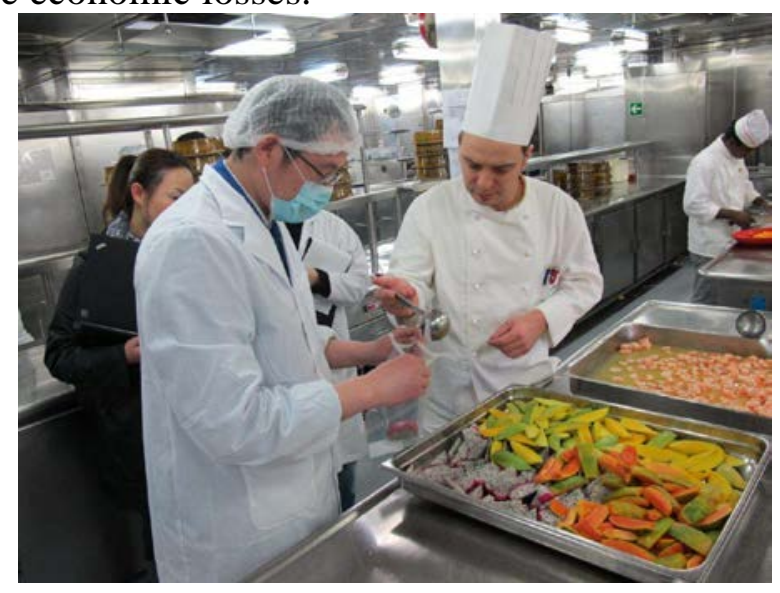

Figure 1 Inspection of processed food

In the traditional food safety inspection, more stay in the breeding environment is sanitary, whether has the epidemic disease infection, whether is the water injection meat and so on, does not pay attention to the veterinary medicine residue in the animal product and the use of the drug feed additive. Amoxicillin, gentamicin, neomycin and clindamycin are the main antibiotics commonly used. The abuse of antibiotics to livestock is very serious. According to statistics, more than $90 \%$ of the antibiotics are used in animals, of which $90 \%$ are used as feed additives to improve feed conversion rate. Surveys and studies have shown that more than half of the demonstration farms don't know much about the ban on penicillin and aren't able to do so in accordance with the veterinary drug withdrawal period for penicillin. There are also drugs that produce milk gas and are forbidden to be used during the laying period, and many farms are not strictly enforced. In the treatment of livestock diseases, they are treated by random means, which may lead to excessive use of drugs or prolonged treatment courses, resulting in the residual veterinary drugs.

\subsection{Poor Quality}

The production, processing, transportation and marketing of animal food products may cause contamination of food for man-made reasons, as well as considering the addition of prohibited drugs, such as residues in the processing process. Because some animal products in the process of processing and storage, people in order to better product appearance, so will use some bleach powder, mirabilite, pigment and so on illegally, these additives will extend the shelf life of the product, some antibiotic use can achieve the effect of sterilization.[4]Because the processing and storage of food is difficult, food materials are prone to corruption, discoloration, poor taste, and even some products are processed and shelf life is too long, which leads to the phenomenon and problems such as the migration of harmful substances and the corruption of internal bags.

For some dairy products, the share of high-quality products in dairy products is extremely limited because of the presence of pesticides, antibiotics and the presence of microorganisms exceeding the standard. Because of the short shelf life and easy acidification of dairy products, in order to cater to the senses of consumers, many merchants will add various flavoring agents, thickeners and so on to the products, but the product label will not be explained. Some egg products 
have been detected in the presence of lead oxide and other illegal substances, some eggs will appear in the lead content is too high, which is due to poor hygiene and manufacturers processing environment.

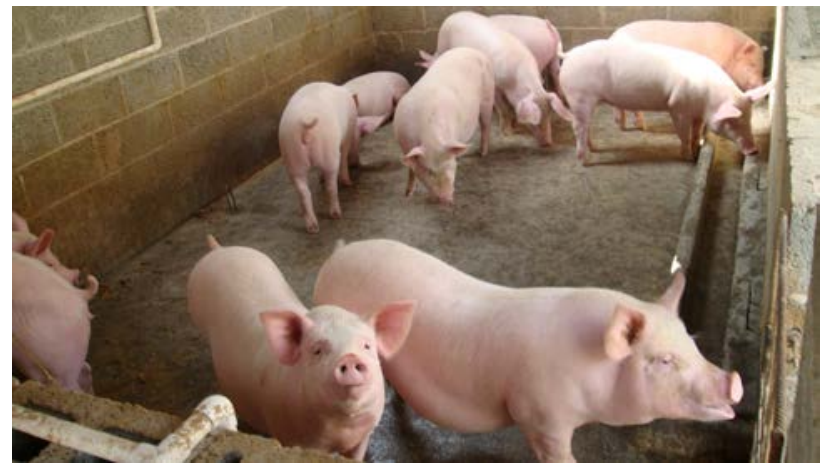

Figure 2 Development of aqua cult ure

\section{Problems in Food Quality and Safety Testing}

In the process of animal food production, many law enforcement departments need to enforce the law in sections, this kind of law enforcement method and law enforcement system will cause the blind spot of possible repeated supervision or supervision, which will lead to the lack of responsibility. Taking lean meat essence as an example, the Ministry of Agriculture's abattoir veterinarians did not pass the quarantine according to law and started with a qualified disease-free certificate and lacked the corresponding legal duty basis. The ministry says lean meat is the head of the agriculture sector, leading to major loopholes in china's regulation of "lean meat" in meat products.

At present, our country also lacks the food safety risk assessment system with the international track, for the food processing production, the storage transportation and so on link will have the processing pollution, the artificial addition contraband and so on risk. In order to avoid the occurrence of this kind of risk, it is necessary to ensure the food safety supervision from beginning to end, to ensure the food safety risk of animal origin, the risk assessment of food safety can be synchronized with the corresponding legal system of the world, and get the recognition of the world, and construct a perfect supervision, inspection and certification system.

\section{Recommendations for Food Quality and Safety Testing Based on Big Data}

\subsection{Deepen the Whole Process of Quality and Safety Backtracking System}

In order to construct a traceable management system and put forward effective food safety measures, we can construct the meat and vegetable circulation traceability system and the animal identification circulation traceability system according to the request of the Ministry of Agriculture. These two management systems are complementary to each other, and in the specific implementation process, the law enforcement authorities need to carry out the law enforcement according to the need for specific circumstances. For animal food safety production management, can be incorporated into the food and drug quality and safety departments to carry out law enforcement. From the technical level, it is necessary to implement the localization of low-cost animal electronic identification, using big data technology to achieve the whole process of data and information management. The first is to carry out a comprehensive traceability system data standardization management, to be able to achieve data collection, transmission, storage, processing and other comprehensive comprehensive technology. Using the idea of combining cloud computing and Internet of things platform, the unified food safety traceability software covering many industries is constructed to realize the system management of different types of enterprise users.

\subsection{Quality and Safety Control Technology of Food Industry Chain}

Using modern sensor technology and Internet technology to build modern intelligent pig farm, it 
can realize the operation of electronic marking, automatic weighing, automatic feeding and so on to the breeding farm, through the electronic tag RFID technology to carry on the individual identification and tracking, realize the whole process tracking record of growth, and play a very important role in the breeding process. For example, in cattle farm, RFID ear electronic tag can be used to record the specific production performance of the cow, including from birth to feeding, epidemic prevention and so on, according to different individual differences to carry out different feeding quantity, while meeting the needs of individual individuals, but also can reduce the cost of feeding. In the feeding process of the cow, it is able to realize its feeding time and amount in the system, whether the cow is in the state of estrus, use the computer system and Internet of things technology to understand the state of estrus of the cow, and understand the health of the cow in real time. For the cow's pregnancy, delivery state and so on can understand and master in detail, do not need to use the traditional paper file to record, query and so on, improve efficiency but also reduce the probability of error.

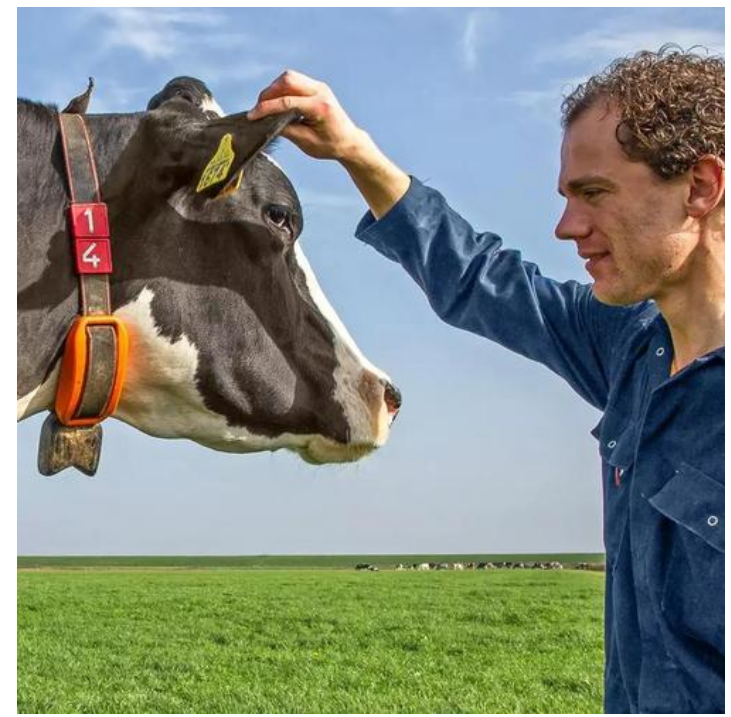

Figure 3 RFID ear labels in the breeding process in breeding process

\section{Conclusion}

The development of Internet and Internet of things technology, can be applied to the quality and safety tracking and detection of modern food, from the initial production, processing, transportation, storage, sales and so on may have the risk of affecting the quality and safety of food, based on big data technology can achieve the supervision of the whole process of food. The use of big data technology can improve the inexperience of food safety supervision in China, improve the detection level of food quality and safety, and promote the healthy and sustainable development of food industry.

\section{References}

[1] Ren, Miaomiao., Shi, Renjie., Shu, Xiaobin. Research on Computer Security Vulnerability Detection Technology in Big Data Background. Computer Knowledge and Technology, vol. 14, no. 21, pp. 61-62, 2017.

[2] Lu, Rigang., Chen, Wei., Su, Hao. Construction of Food and Drug Detection Data Management System Based on Big Data. Chinese Pharmacy, no. 7, pp. 661-665, 2018.

[3] He, Huan., Chen, Qiaoling., Hu, Kang. Research on the Application of Big Data in American Food Safety Supervision and Its Enlightenment to China. Journal of Food Safety and Quality Inspection, vol. 9, no. 10, pp. 267-274, 2017.

[4] Liu, Jinqiu. Shenzhen Inspection and Quarantine Bureau to explore the application of big data 
services import and export food quality information monitoring. China Food, No.659, pp. 79-79, 2014. 